\title{
STABILIZATION OF NEUTRAL SYSTEMS WITH SATURATING INPUTS
}

\author{
J.M. Gomes da Silva Jr. ${ }^{*, 1}$ E. Fridman ${ }^{* *}$ \\ A. Seuret ${ }^{* * *}$ J.P. Richard ${ }^{* * *}$ \\ * UFRGS - Department of Electrical Engineering \\ Av. Osvaldo Aranha 103, 90035-190 Porto Alegre, Brazil. \\ e-mail: jmgomes@eletro.ufrgs.br \\ ** Department of Electrical Engineering-Systems \\ Tel-Aviv University, Tel-Aviv 69978, Israel. \\ e-mail: emilia@eng.tau.ac.il \\ *** LAGIS - Ecole Centrale de Lille, \\ 59651 Villeneuve d'Ascq cedex, France \\ e-mail: seuret.alexandre; jean-pierre.richard@ec-lille.fr
}

\begin{abstract}
This paper focuses on the stabilization problem of neutral systems in the presence of control saturation. Based on a descriptor approach and the use of a modified sector condition, global and local stabilization conditions are derived using Lyapunov-Krasovskii functionals. These conditions allow to consider systems presenting time-varying delays and are formulated directly as linear matrix inequalities (LMIs). Optimization problems are proposed with the aim of computing stabilizing state feedback control laws. Copyright (c) 2005 IFAC
\end{abstract}

Keywords: time delay, saturation, stabilization, stability domains, robustness.

\section{INTRODUCTION}

In the last years great attention has been paid to stability and control of time-delay systems (Niculescu, 2001) (Kolmanovskii and Myshkis, 1999) (Richard, 2003). This is due to the fact that the behavior of many physical systems (mechanical, chemical processes, telecommunication, etc.) can be modeled by functional differential equations. Delays can appear in the state, input or output variables (retarded systems), as well as in the state derivative (neutral systems). Furthermore, it is well known that the presence of the delays in control systems can lead to bad timedomain performances or even to the instability of the closed-loop system. Hence we can find in the literature a great amount of techniques and

\footnotetext{
1 Partially supported by CNPq, Brazil
}

methodologies dealing with the stability and stabilization of time-delay systems (retarded and also neutral), and associated problems such as performance, robustness and filtering.

The difficulty in controlling time-delay systems becomes even greater if the control is forced to be bounded. Unfortunately, this is a practical constraint, which comes from the impossibility of actuators to drive signals with unlimited amplitude or energy to the controlled plants. For retarded systems, some works addressing the stability and stabilization in the presence of saturating control signals can be found in the literature. In (Oucheriah, 1996) and (Niculescu et al., 1996) globally stabilizing control laws are proposed. In (Chen et al., 1988) and (Tissir and Hmamed, 1992), conditions for stability or stabilization are proposed with state feedback and 
sampled state feedback. However, in these papers, the set of admissible initial conditions for which the asymptotic stability is ensured (i.e. the domain of attraction) in the presence of control saturation is not mentioned or explicitly defined. In (Tarbouriech and Gomes da Silva Jr., 2000), (Cao et al., 2002) estimates of the domain of attraction have been found via polytopic models for describing the saturation effects. On the other hand, considering neutral systems, we can cite only (Tarbouriech and Garcia, 1999). In that paper, using a polytopic approach for modeling saturation effects, it is proposed a method for computing stabilizing state feedback controls with the aim of maximizing the set of admissible initial conditions. It should also be pointed out that the method applies only to the case of time-invariant delays, and the conditions (derived in the delay independent context) are presented in the form of nonlinear matrix inequalities. Furthermore, due to the use of a polytopic approach, only local stability can be ensured.

As in (Tarbouriech and Garcia, 1999), this paper is concerned with the stabilization problem of neutral systems in the presence of control saturation. Based on the descriptor approach (Fridman and Shaked, 2002), and the use of a modified sector condition (Tarbouriech et al., 2004), global and local stabilization conditions are derived in a delay dependent context. Differently from (Tarbouriech and Garcia, 1999), these conditions allows to consider systems presenting time-varying delays and are formulated directly as linear matrix inequalities (LMIs). Optimization problems are then formulated with the aim of computing stabilizing state feedback control laws. These optimization problems allow to search the maximal delay bound for which a global stabilizing control law can be found. On the other hand, when only local stabilization is possible (e.g. when the open-loop system is unstable), the optimization objective consists in finding a control law that maximizes an estimate of the domain of attraction.

Notations. Throughout the paper $\Re^{n}$ denotes the $n$ dimensional Euclidean space with vector norm $|\cdot|, A_{i}$ denotes the $i$ th row of matrix $A$. For two symmetric matrices, $A$ and $B, A>B$ means that $A-B$ is positive definite. $A^{\prime}$ denotes the transpose of $A$. $I$ denotes an identity matrix of appropriate order. $\lambda_{\max }(P)$ and $\lambda_{\min }(P)$ denote respectively the maximal and minimal eigenvalues of matrix $P . \mathcal{C}_{h}=\mathcal{C}\left([-h, 0], \Re^{n}\right)$ is the Banach space of continuous vector functions mapping the interval $[-h, 0]$ into $\Re^{n}$ with the norm $\|\phi\|_{c}=\sup _{h \leq t \leq 0}\|\phi(t)\| .\|\cdot\|$ refers to either the Euclidean vector norm or the induced matrix 2-norm. $\mathcal{C}_{h}^{v}$ is the set defined by $\mathcal{C}_{h}^{v}=\left\{\phi \in \mathcal{C}_{h} ;\|\phi\|_{c}<v, v>0\right\}$.

\section{PROBLEM STATEMENT}

Consider the following neutral type linear system:

$$
\begin{gathered}
\dot{x}(t)-F \dot{x}(t-\tau(t))=A x(t)+ \\
A_{d} x(t-\tau(t))+B u(t) \\
x\left(t_{0}+\theta\right)=\phi(\theta), \forall \theta \in[-h, 0], t_{0} \in \Re_{+}, \phi \in \mathcal{C}_{h}^{v},
\end{gathered}
$$

where $x(t) \in \Re^{n}$ and $u(t) \in \Re^{m}$ are respectively the state and the input vectors, $\tau(t)$ corresponds to a time-varying delay that satisfies

$$
0 \leq \tau(t) \leq h, \quad \dot{\tau}(t) \leq d<1
$$

The initial function $\phi(\theta)$ is supposed to be differentiable. Matrices $A, A_{d}, B$ and $F$ are real constant matrices of appropriate dimensions. To apply the Lyapunov stability Theorem (see p.337 in (Kolmanovskii and Myshkis, 1999)) we assume that $\|F\|<1$.

We suppose that the input vector $u$ is subject to amplitude limitations defined as follows:

$$
\left|u_{i}\right| \leq u_{0 i}, \quad u_{0 i}>0, i=1, \ldots, m
$$

Consider now a state feedback control law $u(t)=$ $K x(t)$. Due to the control bounds defined in $(2)$, the effective control signal to be applied to the system is given by $u(t)=\operatorname{sat}(K x(t))$ where $u_{i}(t)=$ $\operatorname{sat}\left(K_{i} x(t)\right)=\operatorname{sign}\left(K_{i} x(t)\right) \min \left\{u_{0 i}, K_{i} x(t)\right\}$

Hence, the closed-loop system reads

$$
\begin{gathered}
\dot{x}(t)-F \dot{x}(t-\tau(t))=A x(t)+A_{d} x(t-\tau(t))+ \\
B \operatorname{Bsat}(K x(t))
\end{gathered}
$$

System (3) will be said globally asymptotically stable if for any initial condition satisfying $\left\|\phi_{x}\right\|_{c} \leq v$ with any finite $v$, the trajectories of the system converge asymptotically to the origin (Oucheriah, 1996), (Niculescu et al., 1996). Similarly to the case of delay-free $(\tau(t)=0)$, the determination of a global stabilizing controller is possible only when some stability assumptions are verified by the open-loop system $(u(t)=0$ ) (Lin and Saberi, 1993). When this hypothesis is not verified, it is only possible to achieve local stabilization. In fact, in the generic case, given a stabilizing matrix $K$, we associate a basin of attraction to the equilibrium point $x_{e}(t) \equiv 0$ of system (5). The basin of attraction corresponds to all initial conditions $\phi(\theta) \in \mathcal{C}_{h}$ such that the corresponding trajectories of system (5) converge asymptotically to the origin. Since the determination of the exact basin of attraction is practically impossible, a problem of interest is to ensure the asymptotic stability for a set of admissible initial conditions $\phi(\theta)$ (Tarbouriech and Gomes da Silva Jr., 2000), (Cao et al., 2002),(Fridman et al., 2003). Of course, this set is included in the basin of attraction. Hence, from the considerations above, in this paper we are interested in study the stabilization problems stated as follows. 
(1) Given $h$ and $d$, find $K$ and a set of admissible initial conditions, as large as possible, for which the stability of the closed-loop system is ensured.

(2) Given $h, d$ and a set of admissible initial conditions, find $K$ such that the asymptotic stability is ensured for all initial conditions of the admissible set.

(3) Maximize the bound on the delay $h$, for which the stability of the closed-loop system can be ensured for some set of admissible initial conditions and a given $d$.

Of course, when it is possible, the objective will be the global stabilization of the closed-loop system. Otherwise, the set of admissible initial conditions will be defined from bounds on $\|\phi(s)\|^{2}$ and $\|\dot{\phi}(s)\|^{2}$. In the sequel, theoretical conditions that allows to address the stabilization problems above are proposed. Based on these conditions, optimization problems will be formulated in the section 4 .

\section{MAIN RESULTS}

Define the following function

$$
\psi(K x(t))=K x(t)-\operatorname{sat}(K x(t))
$$

Note that, $\psi(K x(t))$ corresponds to a decentralized deadzone nonlinearity. Considering the function $\psi(K x(t))$, the closed-loop system can be rewritten as

$$
\begin{aligned}
& \dot{x}(t)-F \dot{x}(t-\tau(t))=(A+B K) x(t)+ \\
& A_{d} x(t-\tau(t))-B \psi(K x(t))
\end{aligned}
$$

Considering a matrix $G \in \Re^{m \times n}$ and defining the following polyhedral set

$\mathcal{S} \triangleq\left\{x \in \Re^{n} ;\left|\left(K_{i}-G_{i}\right) x\right| \leq u_{0 i}, i=1, \ldots, m\right\}(6)$

the following Lemma, concerning the nonlinearity $\psi(K x(t))$ can be stated.

Lemma 1. (Tarbouriech et al., 2004) Consider the function $\psi(K x)$ defined in (4). If $x \in \mathcal{S}$ then the relation

$$
\psi(K x)^{\prime} T[\psi(K x)-G x] \leq 0
$$

is verified for any matrix $T \in \Re^{m \times m}$ diagonal and positive definite.

The result in Lemma 1 can be seen as a generalized sector condition. As will seen in the sequel, differently from the classical sector condition (used for instance in (Tarbouriech et al., 2003)), this condition will allow to obtain stability conditions directly in an LMI form.

Theorem 1. If there exists symmetric positive definite matrices $Q_{1}, H, L, J, X$, matrices $Q_{2}, Q_{3}, Y, W$ and a diagonal matrix $S$ of appropriate dimensions and a scalar $\epsilon$ satisfying

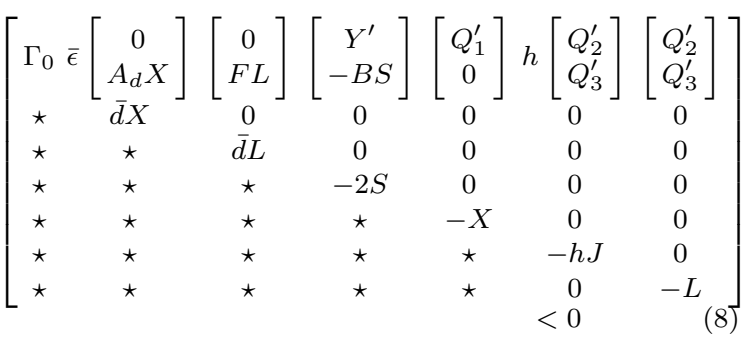

$$
\begin{aligned}
& {\left[\begin{array}{ccc}
J & J\left[\begin{array}{cc}
0 & \epsilon A_{d}^{\prime}
\end{array}\right] \\
\star & H
\end{array}\right] \geq 0} \\
& {\left[\begin{array}{cc}
Q_{1} & (W-Y)_{i}^{\prime} \\
\star & u_{0 i}^{2}
\end{array}\right] \geq 0, \quad i=1, \ldots, m}
\end{aligned}
$$

where $\bar{d}=-(1-d), \bar{\epsilon}=\epsilon-1$,

$\Gamma_{0}=\left[\begin{array}{cc}Q_{2}+Q_{2}^{\prime} & Q_{1}\left(A^{\prime}+\epsilon A_{d}^{\prime}\right)+W^{\prime} B^{\prime}-Q_{2}^{\prime}+Q_{3} \\ \star & -Q_{3}-Q_{3}^{\prime}\end{array}\right]+h H$,

then, for $K=W Q_{1}^{-1}$ and all initial condition satisfying

$$
\begin{aligned}
\delta= & \left(\lambda_{\max }\left(Q_{1}^{-1}\right)+h \lambda_{\max }\left(X^{-1}\right)\right)\|\phi(s)\|^{2} \\
& +\frac{h^{2}}{2} \lambda_{\max }\left(J^{-1}\right)\|\dot{\phi}(s)\|^{2}+h \lambda_{\max }\left(L^{-1}\right)\|\dot{\phi}(s)\|^{2} \leq 1
\end{aligned}
$$

the corresponding trajectories of system (5) converge asymptotically to the origin.

Proof: System (5) is equivalent to the following descriptor system (Fridman and Shaked, 2002):

$$
\begin{gathered}
{\left[\begin{array}{ll}
I & 0 \\
0 & 0
\end{array}\right]\left[\begin{array}{l}
\dot{x}(t) \\
\dot{y}(t)
\end{array}\right]=\left[\begin{array}{rr}
0 & I \\
\left(A+B K+A_{d}\right) & -I
\end{array}\right]\left[\begin{array}{l}
x(t) \\
y(t)
\end{array}\right]+} \\
{\left[\begin{array}{r}
0 \\
F
\end{array}\right] y(t-\tau(t))-\left[\begin{array}{r}
0 \\
A_{d}
\end{array}\right] \int_{t-\tau(t)}^{t} y(s) d s-\left[\begin{array}{c}
0 \\
B
\end{array}\right] \psi(K x(t))}
\end{gathered}
$$

Define now the following matrices: $\bar{x}(t)=\left[\begin{array}{l}x(t) \\ y(t)\end{array}\right]$, $E=\left[\begin{array}{ll}I & 0 \\ 0 & 0\end{array}\right], P=\left[\begin{array}{rr}P_{1} & 0 \\ P_{2} & P_{3}\end{array}\right]=\left[\begin{array}{lr}Q_{1} & 0 \\ Q_{2} & Q_{3}\end{array}\right]^{-1}=Q^{-1}$.

Note that if (8) is satisfied, one has and $-Q_{3}^{\prime}-$ $Q_{3}<0$. Hence, since $Q_{1}>0$, it follows that matrix $Q$ defined above is invertible.

Considering the Lyapunov-Krasovskii functional

$$
\begin{gathered}
V(t)=\bar{x}(t)^{\prime} E P \bar{x}(t)+\int_{t-\tau(t)}^{t} x(s)^{\prime} M x(s) d s+ \\
\int_{t-\tau(t)}^{t} y(s)^{\prime} U y(s) d s+\int_{-h}^{t} \int_{t+\theta}^{t} y(s)^{\prime} R y(s) d s d \theta
\end{gathered}
$$

with $R, M, U>0$, we find $\dot{V}(t)=\bar{x}(t) \mathcal{L} \bar{x}(t)-$ $2 \bar{x}(t)^{\prime} P^{\prime}\left[\begin{array}{c}0 \\ A_{d}\end{array}\right] \int_{t-\tau(t)}^{t} y(s) d s-2 \bar{x}(t)^{\prime} P^{\prime}\left[\begin{array}{l}0 \\ B\end{array}\right] \psi(K x(t))+$ 
$2 \bar{x}(t)^{\prime} P^{\prime}\left[\begin{array}{c}0 \\ F\end{array}\right] y(t-\tau(t))+x(t)^{\prime} M x(t)-x(t-\tau(t))^{\prime}(1-$ d) $M x(t-\tau(t))+y(t)^{\prime} U y(t)-y(t-\tau(t))^{\prime}(1-d) U y(t-$ $\tau(t))+h y(t)^{\prime} R y(t)-\int_{-h}^{0} y(t+\theta)^{\prime} R y(t+\theta) d \theta$ where

$\left.\mathcal{L}=\left[\left(A+B K+A_{d}\right)-I\right]^{0} \quad I\right]^{\prime} P+P^{\prime}\left[\begin{array}{rr}0 & I \\ \left(A+B K+A_{d}\right) & -I\end{array}\right]$

Provided that $\left[\begin{array}{ll}R & N \\ \star & Z\end{array}\right] \geq 0$, one obtains (Moon et al., 2001) that $-2 \bar{x}^{\prime} P^{\prime}\left[\begin{array}{c}0 \\ A_{d}\end{array}\right] \int_{t-\tau(t)}^{t} y(s) d s \leq$ $\int_{t-\tau(t)}^{t}\left[\begin{array}{c}y(s) \\ \bar{x}\end{array}\right]^{\prime}\left[\begin{array}{cc}R & N-\left[\begin{array}{ll}0 & A_{d}^{\prime}\end{array}\right] P \\ \star & Z\end{array}\right]\left[\begin{array}{c}y(s) \\ \bar{x}\end{array}\right] d s \leq$ $\int_{t-h}^{t} y(s)^{\prime} R y(s) d s+2 \int_{t-\tau(t)}^{t} \dot{x}(s)^{\prime}\left(N-\left[\begin{array}{ll}0 & A_{d}^{\prime}\end{array}\right] P\right) \bar{x}(t) d s$ $+h \bar{x}^{\prime} Z \bar{x}=\int_{t-h}^{t} y(s)^{\prime} R y(s) d s+2 x(t)^{\prime}\left(N-\left[\begin{array}{ll}0 & A_{d}^{\prime}\end{array}\right] P\right) \bar{x}(t)-$ $2 x(t-\tau(t))^{\prime}\left(N-\left[\begin{array}{ll}0 & A_{d}^{\prime}\end{array}\right] P\right) \bar{x}(t)+h \bar{x}^{\prime} Z \bar{x}$

Choosing (Fridman and Shaked, 2002) now $N=$ $\epsilon\left[\begin{array}{ll}0 & A_{d}^{\prime}\end{array}\right] P$, it follows that $N-\left[\begin{array}{ll}0 & A_{d}^{\prime}\end{array}\right] P=(\epsilon-$ 1) $\left[\begin{array}{ll}0 & A_{d}^{\prime}\end{array}\right] P$. Considering $K Q_{1}=W$ and $H=$ $Q^{\prime} Z Q$, from the overbounding above and Lemma 1 , provided $x \in \mathcal{S}$, it follows that $\dot{V}(t) \leq \xi^{\prime} \Gamma \xi$, with $\Gamma$ defined as

$$
\left[\begin{array}{cccc}
P^{\prime} \Gamma_{0} P+\Phi \bar{\epsilon} P^{\prime}\left[\begin{array}{c}
0 \\
A_{d}
\end{array}\right] & P^{\prime}\left[\begin{array}{c}
0 \\
F
\end{array}\right]\left[\begin{array}{c}
G^{\prime} T \\
0
\end{array}\right]-P^{\prime}\left[\begin{array}{c}
0 \\
B
\end{array}\right] \\
\star & \overline{d M} & 0 & 0 \\
\star & \star & \bar{d} U & 0 \\
\star & \star & \star & -2 T
\end{array}\right]
$$

where $\Phi=\left[\begin{array}{cc}M & 0 \\ 0 & h R+U\end{array}\right], T$ is a diagonal definite positive matrix, and $\xi=\left[\bar{x}^{\prime} x(t-\tau(t))^{\prime} y(t-\right.$ $\left.\tau(t))^{\prime} \psi(K x(t))^{\prime}\right]^{\prime}$.

Define $\Xi_{1}=\operatorname{diag}\left\{P^{-1}, M^{-1}, U^{-1}, T^{-1}, I, I, I\right\}$. Applying to the term $\Phi$ Schur's complement formula and further pre and post-multiplying the resulting matrix by $\Xi_{1}^{\prime}$ and $\Xi_{1}$ respectively, we find that $\Gamma<0$ is equivalent to (8), with $X=$ $M^{-1}, J=R^{-1}, L=U^{-1}, S=T^{-1}, G=Y P_{1}$. Consider next the block diagonal matrix $\Xi_{2}=$ $\operatorname{diag}\{R, P\}$. Multiplying (9) by $\Xi_{2}^{\prime}$ and $\Xi_{2}$ from the left and from the right, we obtain that (9) is equivalent to $\left[\begin{array}{cc}R & N \\ \star & Z\end{array}\right] \geq 0$. Hence, (8) and (9) imply that $\dot{V}(t)<0$ provided that $x(t) \in \mathcal{S}, t>0$.

Define the ellipsoidal set $\mathcal{E}=\left\{x \in \Re^{n} ; x^{\prime} P_{1} x \leq\right.$ 1 \}, where $P_{1}=Q_{1}^{-1}$. It is easy to see (Tarbouriech and Gomes da Silva Jr., 2000) that (10) implies that $\mathcal{E} \subset \mathcal{S}$ as defined in (6). Suppose now that the initial condition $\phi(s)$ satisfies (11), and conditions (8)-(10) hold. Hence, from the definition of $V(t)$, one has $x(0)^{\prime} P_{1} x(0) \leq V(0) \leq \delta \leq 1$ and, in this case, it follows that $x(0) \in \mathcal{E} \subset \mathcal{S}$ and, as a consequence, $\dot{V}(0)<0$. Then we can conclude that $x(t)^{\prime} P_{1} x(t) \leq V(t) \leq V(0) \leq \delta \leq 1, \quad \forall t \geq 0$, which implies that $x(t) \in \mathcal{S}$. Then, $\dot{V}(t)<0, \forall t>$ 0 for all initial conditions verifying (11).
Theorem 2. If there exists positive definite matrices $Q_{1}, H, L, J, X$, matrices $Q_{2}, Q_{3}, W$ and a diagonal matrix $S$ of appropriate dimensions and a scalar $\epsilon$ satisfying (8)-(9) with $Y=W$, then, for $K=W Q_{1}^{-1}$ system (5) is globally asymptotically stable.

Proof: Consider $G=K$. In this case (7) is verified for all $x \in \Re^{n}$ and the global asymptotic stability follows.

\subsection{Retarded case}

We consider in this section the particular case of system (1) when $F=0$. In this case, we assume that $\tau(t)$ can vary arbitrarily fast, i.e., we focus in the stabilization of the following retarded system

$$
\dot{x}(t)=A x(t)+A_{d} x(t-\tau(t))+B u(t)
$$

with $0 \leq \tau(t) \leq h$.

Theorem 3. If there exists positive definite matrices $Q_{1}, H, J$, matrices $Q_{2}, Q_{3}, Y, W$ and a diagonal matrix $S$ of appropriate dimensions satisfying the following linear matrix inequalities

$$
\begin{gathered}
{\left[\begin{array}{ccc}
\Gamma_{0}+h H & {\left[\begin{array}{c}
Y^{\prime} \\
-B S
\end{array}\right]} & h \\
\star & -2 S & {\left[\begin{array}{l}
Q_{2}^{\prime} \\
Q_{3}^{\prime}
\end{array}\right]} \\
\star & \star & 0 \\
\star & -h J
\end{array}\right]<0} \\
{\left[\begin{array}{ccc}
J & J\left[\begin{array}{ll}
0 & A_{d}^{\prime}
\end{array}\right] \\
\star & H
\end{array}\right] \geq 0} \\
{\left[\begin{array}{ccc}
Q_{1} & (W-Y)_{i}^{\prime} \\
\star & u_{0 i}^{2}
\end{array}\right] \geq 0, \quad i=1, \ldots, m}
\end{gathered}
$$

$\Gamma_{0}=\left[\begin{array}{cc}Q_{2}+Q_{2}^{\prime} & Q_{1}\left(A+A_{d}\right)^{\prime}+W^{\prime} B^{\prime}-Q_{2}^{\prime}+Q_{3} \\ \star & -Q_{3}-Q_{3}^{\prime}\end{array}\right]$, then, for $K=W Q_{1}^{-1}$ and all initial condition satisfying

$\lambda_{\max }\left(Q_{1}^{-1}\right)\|\phi(s)\|^{2}+\frac{h^{2}}{2} \lambda_{\max }\left(J^{-1}\right)\|\dot{\phi}(s)\|^{2} \leq 1$

the corresponding trajectories of system (14) converge asymptotically to the origin.

Proof: Considering the following Lyapunov-Krasovskii functional

$$
V(t)=\bar{x}(t)^{\prime} E P \bar{x}(t)+\int_{-h}^{0} \int_{t+\theta}^{t} y(s)^{\prime} R y(s) d s d \theta
$$

with $R>0$ and $U>0$, it suffices to follow the same steps as in the proof of Theorem 1 with $\epsilon=1$. 
Theorem 4. If there exists positive definite matrices $Q_{1}, H, J$, matrices $Q_{2}, Q_{3}, W$ and a diagonal matrix $S$ of appropriate dimensions satisfying the linear matrix inequalities (15)-(16) with $Y=W$, then $K=W Q_{1}^{-1}$ is such that system (14) is globally asymptotically stable.

Remark 1. The extension of the conditions in order to consider uncertain systems described by polytopic uncertainties is straightforward. Since the LMIs in Theorems 1, 2 3, 4 are affine in the system matrices $A, A_{d}, B$ and $F$, it suffices to verify conditions of the Theorems for each vertex of the matrix polytope. Furthermore, note that for each vertex, different matrices $Q_{3}$ and $Q_{2}$ can be considered.

\section{OPTIMIZATION PROBLEMS}

\subsection{Maximization of the delay for which global stability is ensured}

In the case where the system can be globally asymptotically stabilizable in the absence of the delays, an interesting problem consists in finding the maximal bound $h^{\star}$ on the time-varying delay $\tau(t)$, for which system (5) can be globally stabilizable. This can be accomplished by solving

$$
\max h
$$$$
\text { subject to (8) and (9) with } Y=W
$$

Due to the product between $h$ and the variables $Q_{2}, Q_{3}, H$ and $J$, the solution of this problem can be obtained by iteratively increasing $h$ until LMIs (8) and (9) become unfeasible.

\subsection{Maximization of the set of admissible initial conditions}

Given $h$ and $d$, in order to ensure the stability of system (5) for by using the Theorem 1, the admissible initial conditions must verify condition (11). Assume that $\|\phi(s)\|^{2}=\delta_{1}$ and $\|\dot{\phi}(s)\|^{2}=\delta_{2}$. In this case, a synthesis objective can be the maximization of the admissible bounds $\delta_{1}$ and $\delta_{2}$. This problem can be addressed as follows.

Note that smaller are the maximal eigenvalues of $Q_{1}^{-1}, X^{-1}, J^{-1}$ and $L^{-1}$, larger can be $\delta_{1}$ and $\delta_{2}$ for which (11) is verified. Consider then the following auxiliary LMIs

$$
\begin{gathered}
{\left[\begin{array}{cc}
\lambda_{Q 1} I & I \\
\star & Q_{1}
\end{array}\right] \geq 0,\left[\begin{array}{cc}
\lambda_{X} I & I \\
\star & X
\end{array}\right] \geq 0,} \\
{\left[\begin{array}{cc}
\lambda_{J} I & I \\
\star & J
\end{array}\right] \geq 0,\left[\begin{array}{cc}
\lambda_{L} I & I \\
\star & L
\end{array}\right] \geq 0}
\end{gathered}
$$

it follows that these LMIs are respectively equivalent to $\lambda_{Q 1} \geq \lambda_{\max }\left(Q_{1}^{-1}\right), \lambda_{X} \geq \lambda_{\max }\left(X^{-1}\right)$, $\lambda_{J} \geq \lambda_{\max }\left(J^{-1}\right)$ and $\lambda_{L} \geq \lambda_{\max }\left(L^{-1}\right)$
In this case the following optimization problem can be considered:

$$
\begin{gathered}
\min \beta_{1} \lambda_{Q 1}+\beta_{2} \lambda_{X}+\beta_{3} \lambda_{J}+\beta_{4} \lambda_{L} \\
\text { subject to } \\
\text { (8), (9), (10) and (21) }
\end{gathered}
$$

where $\beta_{1}, \beta_{2}, \beta_{3}$ and $\beta_{4}$ are weights that should be tuned in order to satisfy some trade-off between $\delta_{1}$ and $\delta_{2}$.

\subsection{Maximizations for a given set of admissible initial conditions}

Consider now $\delta_{1}>0$ and $\delta_{2}>0$. The idea is then to compute $K$ in order to guarantee the stability for all initial conditions satisfying $\|\phi(s)\|^{2} \leq \delta_{1}$ and $\|\dot{\phi}(s)\|^{2} \leq \delta_{2}$. This case can be addressed considering the auxiliary LMIs (21) and the following additional constraint:

$$
\left(\lambda_{Q 1}+h \lambda_{X}\right) \delta_{1}+\left(0.5 h^{2} \lambda_{J}+h \lambda_{L}\right) \delta_{2}-1 \leq 0(23
$$

In this case, for instance, the following optimization criteria can be used:

- maximize the bound $h$ for which is possible to find a stabilizing gain.

- maximize the decay rate in the linearity region of the closed-loop system (Tarbouriech and Gomes da Silva Jr., 2000)

- minimize an upper bound to a given cost function (guaranteed cost problem)

\section{NUMERICAL EXAMPLES}

Example 1. Consider system (1) with

$$
\begin{gathered}
A=\left[\begin{array}{cc}
-3 & 1 \\
0 & -4
\end{array}\right] ; A_{d}=\left[\begin{array}{cc}
0.5 & 0 \\
0.5 & -0.5
\end{array}\right] ; B=\left[\begin{array}{l}
1 \\
0
\end{array}\right] \\
F=\left[\begin{array}{cc}
0.5 & 0 \\
0 & 0.5
\end{array}\right] ; d=0.5 ; h=1
\end{gathered}
$$

From Theorem 2, taking $\epsilon=0.1$, it is then possible to ensure the global asymptotic stability (GAS) of the system with, for instance, $K=[-0.138-$ 0.381]. Furthermore, it is possible to ensure the GAS for $h \leq 23 \times 10^{3}$ and $d \leq 0.6$. Hence, in this case, the limit on the delay variation is more critical.

Example 2. Consider system (1) with

$$
\begin{gathered}
A=\left[\begin{array}{cc}
1 & 1.5 \\
0.3 & -2
\end{array}\right] ; A d=\left[\begin{array}{cc}
0 & -1 \\
0 & 0
\end{array}\right] ; B=\left[\begin{array}{c}
10 \\
1
\end{array}\right] \\
F=\left[\begin{array}{cc}
0.2 & 0 \\
0 & 0.2
\end{array}\right] ; u_{0}=15 ; h=1 ; d=0.1
\end{gathered}
$$


Considering the optimization problem (22) with $\beta_{1}=\beta_{2}=\beta_{3}=\beta_{4}=1$, the following gain is obtained: $K=[-0.278-0.139]$. This gain ensures the asymptotic stability for any $\|\phi(s)\|,\|\dot{\phi}(s)\|$ satisfying:

$$
51 \times 10^{-4}\|\phi(s)\|^{2}+9.34 \times 10^{-4}\|\dot{\phi}(s)\|^{2} \leq 1
$$

Hence, for instance, the stability is ensured for $\|\phi(s)\|=\|\dot{\phi}(s)\|<12.88$. As expected, for a greater value of the upperbound of the delay, $h=2$, ones obtains with the gain $K=[-0.248-$ 0.136 ] a smaller set of stability (obtained with $\epsilon=0.11):\|\phi(s)\|=\|\dot{\phi}(s)\|<2.21$.

On the other hand, if $F=0$ (i.e. retarded system), it is possible to ensure the asymptotic stability of initial conditions satisfying $\|\phi(s)\|=\|\dot{\phi}(s)\|<$ 79.546 with the gain $K=\left[\begin{array}{ll}-7.005 & 0.652\end{array}\right]$. Note that the bound on the admissible conditions is slightly larger than the ones obtained in (Fridman et al., 2003) (79.43), (Cao et al., 2002) (58.40). It should however be highlighted that in our case the delay can vary arbitrarily fast and the number of LMIs to test is lower. These facts indicates that the proposed method is potentially less conservative than the previous approaches.

Considering now the problem described in section 4.3 , if the initial conditions are supposed to verify $\|\phi(s)\|^{2}=\|\dot{\phi}(s)\|^{2}<50$, the state feedback gain $K=\left[\begin{array}{ll}-0.524 & 0.867\end{array}\right]$ asymptotically stabilizes the system for all time variant delays bounded by $h_{\max }=7.015$

\section{CONCLUDING REMARKS}

The synthesis of stabilizing gains for linear neutral systems in the presence of saturating inputs and time-varying delays have been addressed. Both global and local stabilization conditions have been derived. Convex optimization problems, with LMI constraints, have then been proposed in order to compute the stabilizing gains and the associated sets of admissible initial conditions for the closedloop system. The extension of the results to uncertain polytopic systems is straightforward.

\section{REFERENCES}

Cao, Y., Z. Lin and T. Hu (2002). Stability analysis of linear time-delay systems subject to input saturation. IEEE Trans. on Circ. and Syst. I 49, 233-240.

Chen, B. S., S. S. Wang and H. C. Lu (1988). Stabilization of time-delay systems containing saturating actuators. Int. J. of Contr. 47, 867-881.
Fridman, E., A. Pila and U. Shaked (2003). Regional stabilization and $h_{\infty}$ control of timedelay systems with saturating actuators. Int. J. of Rob. and Nonlin. Contr. 13, 885-907.

Fridman, E. and U. Shaked (2002). An improved stabilization method for linear timedelay systems. IEEE Trans. Autom. Contr. 47(11), 1931-1937.

Kolmanovskii, V. and A. Myshkis (1999). Applied Theory of functional differential equations. Kluwer.

Lin, Z. and A. Saberi (1993). Semi-global exponential stabilization of linear systems subject to input saturation via linear feedback. Syst. 8 Contr. Lett. 21, 225-239.

Moon, Y.S., P. Park, W.H. Kwon and Y.S. Lee (2001). Delay-dependent robust stabilization of uncertain state delayed systems. Int. J. of Contr. 74, 1447-1455.

Niculescu, S-I. (2001). Delay Effects on Stability. A Robust Control Approach. Springer-Verlag. Berlin, Germany.

Niculescu, S-I., J. M. Dion and L. Dugard (1996). Robust stabilization for uncertain timedelay systems containing saturating actuators. IEEE Trans. Autom. Contr. 41(5), 742747 .

Oucheriah, S. (1996). Global stabilization of a class of linear continuous time delay systems with saturating controls. IEEE Trans. on Circ. and Syst. I 43(12), 1012-1015.

Richard, J.P. (2003). Time-delay systems: an overview of some recent advances and open problems. Automatica 39, 1667-1604.

Tarbouriech, S. and G. Garcia (1999). Stabilization of neutral linear time-delay systems with saturating actuators. In: Proc. of the 38th IEEE Conf. on Dec. and Contr.. pp. 20112016.

Tarbouriech, S. and J. M. Gomes da Silva Jr. (2000). Synthesis of controllers for continuous-time delay systems with saturating controls via LMIs. IEEE Trans. Autom. Contr. 45(1), 105-111.

Tarbouriech, S., J. M. Gomes da Silva Jr. and G. Garcia (2004). Delay-dependent antiwindup strategy for linear systems with saturating inputs and delayed outputs. Int. J. of Rob. and Nonlin. Contr. 14, 665-682.

Tarbouriech, S., J.M. Gomes da Silva Jr. and G. Garcia (2003). Delay-dependent antiwindup loops for enlarging the stability region of time-delay systems with saturating inputs. Trans. ASME - J. of Dyn. Syst., Meas.and Contr. 125(1), 265-267.

Tissir, E. and A. Hmamed (1992). Further results on the stabilization of time delay systems containing saturating actuators. Int. J. of Syst. Sci. 23, 615-622. 